\title{
Effects of Wavelength Variation on Localized Photoemission in Triangular Gold Antennas
}

\author{
Christopher M. Scheffler ${ }^{1 *}$, Robert C. Word ${ }^{1}$, Rolf Könenkamp ${ }^{1}$ \\ 1. Portland State University, Department of Physics, Portland, Oregon, USA. \\ * Corresponding author: schef@pdx.edu
}

Exposing metal-dielectric structures to light can result in surface plasmon excitation and propagation along the transition interface, creating a surface plasmon polariton (SPP) response. Photoemission electron microscopy (PEEM) has been used to image nanometer scale plasmonic responses in micronsized plasmonic devices [1 - 2]. With PEEM, optical responses can be characterized in detail, aiding in the development of new types of plasmonic structures and their applications. We show here that in thin, triangular gold platelets SPPs can be excited and concentrated within specific regions of the material. In this regard, the platelets act as receiver antennas by converting the incident light into localized excitations in specific regions of the gold platelets. The excited areas can be significantly smaller than the wavelength of the incident light $(\lambda \leq 1 \mu \mathrm{m})$. By varying the wavelength of the light, the brightness of the excited spots can be changed, and the electron emission can effectively be switched on or off for a specific region. These experimental findings are directly observed in PEEM imaging and are quantitatively evaluated using a finite-element method (FEM).

Our lab has a home-built aberration-corrected PEEM with a best demonstrated resolution of $5 \mathrm{~nm}$ [3]. The microscope utilizes a Spectra-Physics Ti:Sapphire laser with a fundamental wavelength in the range of $780-920 \mathrm{~nm}$. Sample illumination is achieved from above at $60^{\circ}$ to the surface normal or from below at normal incidence. The samples consist of thin, triangular gold platelets deposited onto indium tin oxide coated borosilicate glass. The gold platelets are grown in a wet-chemical technique [4] and are single-crystalline in a $<1 \quad 1 \quad 1>$ orientation. The triangles are approximately equilateral with most measuring between $3-5 \mu \mathrm{m}$ in length and have thicknesses around $50-100 \mathrm{~nm}$. Using COMSOL Multiphysics, the experimental PEEM setup and the sample details are defined in 3D models. The simulation uses a scattering boundary condition and a planar wave approximation for the illumination source. The time-averaged electromagnetic field distribution in the model is calculated and compared to micrographs obtained from PEEM. These micrographs display the time-averaged photoemission yield (Y $\mathrm{PE}_{\mathrm{PE}}$, which depends on the simulated electric field as $\mathrm{Y}_{\mathrm{PE}} \propto|\mathrm{E}|^{2 \mathrm{n}}$, where $\mathrm{n}$ is the number of photons required for a single photoemission event (Figure 1). Our results indicate that in the wavelength range of $600 \mathrm{~nm}<\lambda<800 \mathrm{~nm}$, the electric field strength at the (rear) tip of the gold platelets changes by a factor of $\sim 8$, resulting in an intensity change of $>60$ (Figure 2 ) for single photon excitation processes and a factor of $>10^{3}$ for 2-photon excitation.

In conclusion, we will discuss electron microscopy approaches for characterizing and controlling micron-sized platelet antennas. We show that wavelength change is a feasible and effective mechanism for controlling the localized field intensity as well as the photoemission yield. The observed wavelength dependence may eventually open the door to wavelength modulation control for optical antennas or nano-sized electron sources. 


\section{References}

[1] C. M. Scheffler, R. C. Word, R. Könenkamp, Nanotech. Materials \& Devices Conf. Proceed. (2018).

[2] R. C. Word, R. Könenkamp, Ultramicroscopy 183 (2017), p. 1139 - 1351.

[3] R. Könenkamp et al, Ultramicroscopy 110 (2010), p. 899 - 902.

[4] Z. Guo et al, Colloids and Surfaces A: Physicochem. Eng. Aspects 278 (2006), p. 33 - 38.

[5] This research was supported in part by the US Department of Energy's Basic Science Office under Contract No. DE-FG02-13ER46406 and by the US Department of Defense's Science, Mathematics and Research for Transformation (SMART) Scholarship for Service Program. C.M.S. would like to thank Theodore Stenmark of PSU for providing guidance on COMSOL modeling, PEEM, and plasmonics.

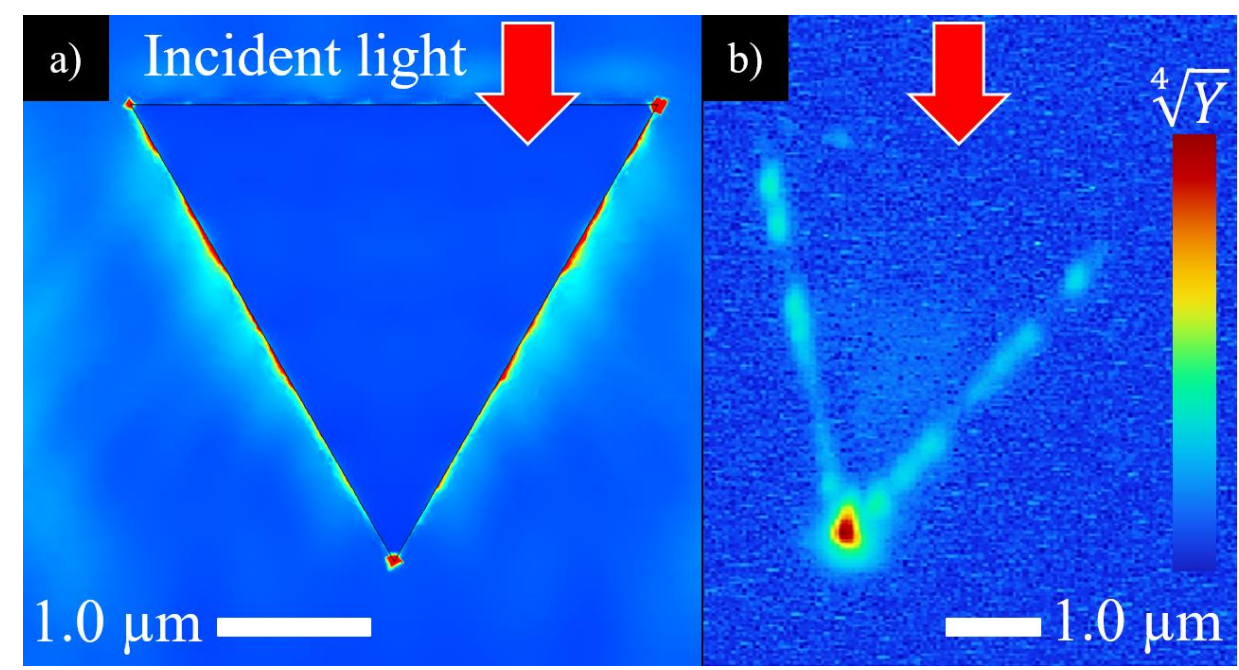

Figure 1. (a) Simulation results of the time-averaged $|E|^{2}$ at the triangular gold surface for $800 \mathrm{~nm}$ light with oblique incidence $\left(\theta=60^{\circ}\right)$ and TM polarization; (b) PEEM micrograph (false color) with the same conditions. Note: The PEEM micrograph (b) is presented as the $4^{\text {th }}$ root of the photoemission yield.

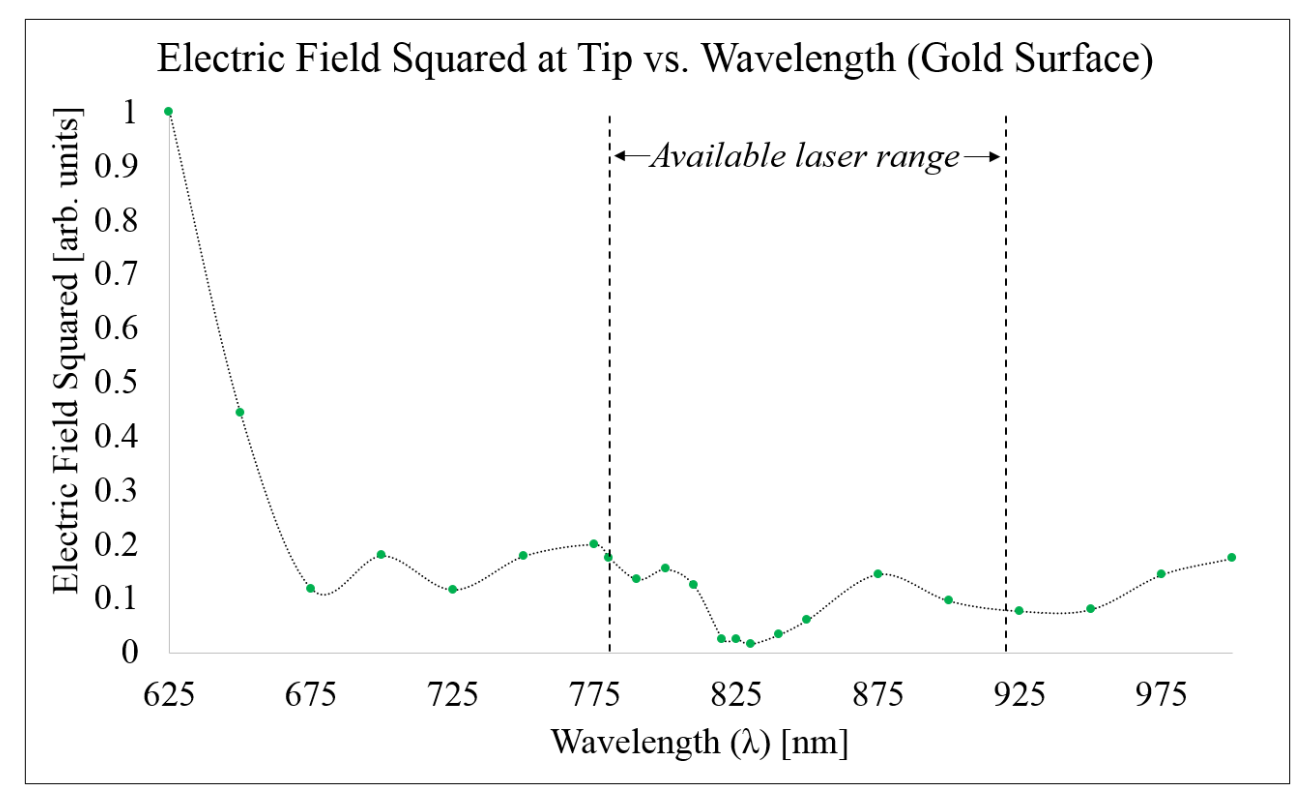

Figure 2. Graph of the simulated time-averaged $|E|^{2}$ at the gold surface of the rear tip vs. wavelength for a $3 \mu \mathrm{m}$ long gold platelet exposed to obliquely incident light $\left(\theta=60^{\circ}\right)$ with $\mathrm{TM}$ polarization for $625 \mathrm{~nm}<\lambda<1 \mu \mathrm{m}$. The fundamental wavelength range of the PEEM laser is indicated by dotted lines. 